\title{
Severity of respiratory disease is correlated with time of first oral feeding and need for a gastrostomy tube at discharge in premature infants born at $<30$ weeks of gestation
}

\author{
Emily Anderson ${ }^{1}$, Matthew Gregoski ${ }^{2}$, Daniel Gehle ${ }^{3}$, William Head ${ }^{3}$, Thomas Hardy ${ }^{2}$, \\ Alison Chapman ${ }^{2}$, and Rita Ryan ${ }^{4}$ \\ ${ }^{1}$ Augusta University and University of Georgia Medical Partnership \\ ${ }^{2}$ Medical University of South Carolina \\ ${ }^{3}$ Medical University of South Carolina College of Medicine \\ ${ }^{4}$ Case Western Reserve University Hospital
}

June 24, 2021

\begin{abstract}
Background: Premature infants who cannot achieve full oral feeds may need a gastrostomy tube (GT) to be discharged from the neonatal intensive care unit (NICU). We previously developed a model to predict which infants born $<30$ weeks (w) gestational age (GA) will require a GT before discharge. Here we report the detailed respiratory variable data to describe the general respiratory course for infants in the NICU $<30 \mathrm{w}$ GA at birth and the association between different levels of respiratory support with postmenstrual age (PMA) at the time of first oral feeding attempt (PMAff), including later need for GT for discharge. Methods: Retrospective chart review of 391 NICU admissions comprising test (2015-2016) and validation (2017-2018) cohorts. Data, including respiratory support, were collected on 204 infants, 41 GT and 163 non-GT, in the test cohort, and 187 infants, 37 GT and 150 non-GT, in the validation cohort. Results: Respiratory data were significantly different between GT and non-GT infants. Infants who required GT for discharge were on significantly higher respiratory support at 30 days of age, 32w PMA, and 36w PMA. Respiratory parameters were highly correlated with PMAff. Conclusion: Respiratory status predicts PMAff, which was the variable in our previously described model that was most predictive of failure to achieve full oral feeing. These data provide a catalyst to develop strategies for improving oral feeding outcome for infants requiring prolonged respiratory support in the NICU.
\end{abstract}

Severity of respiratory disease is correlated with time of first oral feeding and need for a gastrostomy tube at discharge in premature infants born at $<30$ weeks of gestation

Emily Anderson ${ }^{1}$, Mathew J Gregoski ${ }^{2}$, Daniel Gehle ${ }^{3}$, William Taylor Head ${ }^{3}$, K. Thomas Hardy ${ }^{4}$, Alison Chapman $^{4}$, Rita M. Ryan ${ }^{4,5}$

${ }^{1}$ Augusta University Medical College of Georgia, AU/UGA Medical Partnership, Athens, Georgia

${ }^{2}$ Department of Public Health Sciences, Medical University of South Carolina, Charleston, SC

${ }^{3}$ Medical University of South Carolina School of Medicine, Charleston, SC

${ }^{4}$ Department of Pediatrics (Neonatology), Medical University of South Carolina Children's Hospital, Charleston, SC

${ }^{5}$ Department of Pediatrics (Neonatology), Case Western Reserve University, Rainbow Babies \& Children's Hospital, Cleveland, $\mathrm{OH}$ 


\section{Corresponding Author:}

Rita M. Ryan, MD

Rainbow Babies \& Children's Hospital

11100 Euclid Avenue

Cleveland, $\mathrm{OH} 44106$

Telephone: (216) 844-3387

Fax: 216-844-3380

Email:Rita.Ryan@UHhospitals.org

Keywords: prematurity, neonatal intensive care unit, gastrostomy, discharge

Abbreviated Title: Respiratory disease is correlated with oral feeds

\section{Abstract:}

Background : Premature infants who cannot achieve full oral feeds may need a gastrostomy tube (GT) to be discharged from the neonatal intensive care unit (NICU). We previously developed a model to predict which infants born $<30$ weeks (w) gestational age (GA) will require a GT before discharge. Here we report the detailed respiratory variable data to describe the general respiratory course for infants in the NICU $<30 \mathrm{w}$ GA at birth and the association between different levels of respiratory support with postmenstrual age (PMA) at the time of first oral feeding attempt (PMAff), including later need for GT for discharge.

Methods : Retrospective chart review of 391 NICU admissions comprising test (2015-2016) and validation (2017-2018) cohorts. Data, including respiratory support, were collected on 204 infants, 41 GT and 163 non-GT, in the test cohort, and 187 infants, 37 GT and 150 non-GT, in the validation cohort.

Results : Respiratory data were significantly different between GT and non-GT infants. Infants who required GT for discharge were on significantly higher respiratory support at 30 days of age, 32w PMA and 36w PMA. Respiratory parameters were highly correlated with PMAff.

Conclusion : Respiratory status predicts PMAff, which was the variable in our previously described model that was most predictive of failure to achieve full oral feeing. These data provide a catalyst to develop strategies for improving oral feeding outcome for infants requiring prolonged respiratory support in the NICU.

\section{Introduction:}

Very preterm infants experience high rates of medical complications and frequently have prolonged hospitalizations associated with the inability to achieve full oral feedings, often necessitating a gastrotomy tube (GT) or other form of feeding tube to be placed prior to discharge. ${ }^{1}$ It is the practice at our institution to surgically place a GT for secure home feeding in infants unable to attain full oral feeds. To facilitate earlier GT placement and expedite discharge home, we developed a model to identify infants at highest risk for needing a GT. In the process of developing this predictive model, several respiratory variables were identified as being associated with the inability to obtain full oral feeding prior to discharge. Despite many respiratory variables collected in the original cohort, exposure to high frequency ventilation (HFV) was the only respiratory variable that remained independently in the final predictive model. We aim to demonstrate the respiratory course of infants born at $<30 \mathrm{w}$ GA, and how these data are associated with the ability to obtain full oral feeding. It has previously been suspected that positive pressure support in infants can interfere with the development of the swallowing reflex needed to obtain full oral feeds. ${ }^{2,3}$ In addition, since postmenstrual age (PMA) at the time of first oral feeding (PMAff) was the single most predictive factor in 
attaining full oral feeding prior to NICU discharge, we wanted to test the hypothesis that respiratory status predicts PMAff.

\section{Methods:}

The two-year test cohort consisted of 204 infants born $<30 \mathrm{w}$ GA admitted to the NICU at the Medical University of South Carolina in 2015 and 2016. The cut-off of 30w GA was derived from earlier work that eliminated infants who received a GT for non-prematurity-related reasons, such as congenital anomalies. ${ }^{4}$ Infants who died before discharge or who were admitted after 7 days of age were excluded. We collected retrospective data including demographics, feeding, and other disease variables which are reported elsewhere. ${ }^{4}$ Respiratory data were collected at three time points in the original cohort: 30 days (d) of age, $32 \mathrm{w}$ PMA and 36w PMA. Variables included $\mathrm{F}_{\mathrm{i}} \mathrm{O} 2$ and mode of respiratory support: none, low-flow nasal cannula (NC), heated humidified high-flow NC (HFNC), continuous positive airway pressure (CPAP), nasal intermittent positive pressure ventilation (NIPPV), conventional ventilation (CV) or high frequency jet or oscillatory ventilation (HFV), used as a rescue measure. We also collected total ventilator days, total ventilator + CPAP days, PMA at last ventilator day, PMA at last CPAP day, and PMAff. Some infants never had an oral feeding attempt due to severity of illness or need for tracheostomy; for this data to contribute to the model variable, these infants were arbitrarily assigned a PMAff of $52 \mathrm{w}$, which was one week beyond the highest PMAff found for all GT infants $(51 \mathrm{w})$.

The validation cohort consisted of 187 infants born $<30 \mathrm{w}$ GA admitted to the NICU at the Medical University of South Carolina from 2017-2018. We recorded the same demographic data for each cohort. In the original cohort, respiratory mode at $32 \mathrm{w}$ PMA was the most predictive timepoint for respiratory parameters to predict GT outcome, so respiratory variables were only recorded at $32 \mathrm{w}$ PMA in the validation cohort. Any exposure to HFV was also again recorded. In the original model development, we did not look specifically at bronchopulmonary dysplasia (BPD). BPD diagnoses were retroactively assigned to infants in the original cohort using the Shennan et al definition of "oxygen use at 36 weeks PMA." ${ }^{5}$ This data is not available in the original model report.

These data were collected with approval of the MUSC Institutional Human Subjects Review Board. Normally distributed continuous data are presented as mean \pm standard deviation and $\mathrm{P}$-value derived by t-test; non-normally distributed continuous data are presented as median, interquartile range and compared with Wilcoxon rank sum test; categorical data are presented as number (percent) and tested by chi-squared test. A 2 (GT) by 3 (Fio2) repeated measures ANOVA was utilized to examine Fi02 rates by GT status over the time periods of 30 days, 32 weeks, and 36 weeks. P-values of $<0.05$ were considered significant. Pearson's coefficient was used to test for correlation between respiratory variables and PMAff. Univariate analyses were performed using STATA (State College, TX) and SPSS version 25 (Armonk, NY: IBM Corp). Some figures were created using SPSS version 25.

\section{Results:}

In the original cohort of 204 infants, 41 needed GT placement before discharge. In the validation cohort of 187 infants, 37 needed GT placement. Respiratory parameters were highly associated with need for GT at discharge in the original test cohort (Table 1) and the validation cohort (Table 2).

We examined both a broader categorization of respiratory support defined as high support (CPAP, NIPPV, CV, HFV) vs. low support (HFNC, LFNC, no support) (Figure 3), as well as the more detailed distribution of respiratory mode (Figure 4). When examined in more detail, the difference in respiratory support in the two groups (GT vs. non-GT) is quite striking. Infants who failed to achieve full oral feeding were on significantly more respiratory support, as well as higher oxygen (Figure3), throughout the NICU course. Both FiO2 and the level of respiratory support do improve over time regardless of GT outcome (Figure 1). Every variable examining duration of continuous respiratory support, including total ventilator days, total ventilator + CPAP days, PMA at last ventilator day, and PMA at last CPAP day (Figure 4), were significantly different between the GT and non-GT infants. 
We also looked specifically at need for HFV and GT status. At MUSC, HFV is used as a rescue mode after failure to achieve adequate ventilation and/or oxygenation on conventional ventilator support; hence, infants exposed to HFV at some point during their NICU course would have had significantly higher respiratory support needs at that time. In both the original and validation cohorts, infants who later received a GT were significantly more likely to have some exposure to HFV $(\mathrm{P}<0.0001)$.

Given the large differences between respiratory variables in GT and non-GT infants, we also examined the association between respiratory support and PMAff (Figure 2). In the original test cohort for the predictive model, infants who received a GT had their first oral feeding attempt at 40.3w PMA while the non-GT infants' PMAff on average was 33.6 weeks. ${ }^{6}$ All variables measuring duration of respiratory support were also highly correlated with PMAff with $\mathrm{r}$ values of $0.642-0.884$ and with $\mathrm{p}$ values all $<0.001$.

To further investigate an additional pulmonary complication seen in the NICU, we examined the incidence of bronchopulmonary dysplasia (BPD) between GT and non-GT infants in the original test cohort (Table 1).

Discussion:

The respiratory course of premature infants in the NICU plays a significant role in determining oral feeding success. Preterm infants $<30 \mathrm{w}$ GA are at increased risk for pulmonary complications, especially BPD. ${ }^{7,8}$ BPD can lead to higher respiratory support requirements, delaying the initiation of oral feeding attempts. ${ }^{9}$ Gianni et al demonstrated that PMA at the time of achieving full oral feeding was significantly earlier as gestational age or birth weight increased. BPD was significantly associated with an older PMA at full oral feeding. ${ }^{8}$ Our data supports this conclusion as well. We showed that BPD is associated with an older PMAff, the most important variable in our original model describing risk for GT placement. ${ }^{4}$

Premature infants do not have mature suck-swallow-breathe coordination, limiting their ability to orally feed. Preterm infants are prone to initiate swallowing at unsafe phases of the respiratory cycle, increasing the risk of desaturation and aspiration. ${ }^{10}$ The earliest successful synchronization of sucking, swallowing, respiration and esophageal function has been reported to occur in premature infants sometime between $32-34 \mathrm{w}$ PMA. ${ }^{11}$ The development of this coordination is further delayed in premature infants with BPD, who often require higher levels of respiratory support for longer periods of time. ${ }^{12,13}$ Positive pressure support has been suspected to inhibit closure of the larynx and interfere with the swallowing reflex. ${ }^{2,3}$ Infants on nasal CPAP have been shown to have increased risk of aspiration during oral feeds, likely due to interfering signals from the positive pressure. $^{11}$

In our original model development, although every respiratory variable was associated with the GT/no GT outcome in univariate analysis, only exposure to HFV remained in the final model. ${ }^{4}$ We suspected that respiratory status is highly correlated to the most significant variable in the model, PMAff, and now provide evidence supporting that hypothesis. However, this conclusion may be confounded because there is variability amongst NICUs regarding when infants may or may not receive oral feeding attempts. ${ }^{14}$ For instance, our NICU policy restricted oral feeding attempts to infants who were on nasal cannula flow $<0.5$ LPM. This fact provides an interesting area for further study. Additionally, this suggests we should be more thoughtful about when we introduce or withhold oral feeding for infants requiring high respiratory support during their NICU course. There are likely critical periods of neurodevelopment during which we need to engage neural and muscular pathways for oral feeding; if this window of time is missed, it may become much more difficult for later development of this skill. ${ }^{15}$

We noted a significant gender difference in infants requiring a GT (63\% male) versus infants not requiring a GT ( $45 \%$ male) in the original test cohort. This was significant in the test cohort on univariate analysis but did not remain in the multivariate model. In the separate validation cohort, this was no longer significant ( $57 \%$ vs. $49 \%)$. However, when the two cohorts are combined, the p value is again significant: $47 / 78(60 \%)$ males in the GT infants, vs. 148/313 (47\%) in the non-GT cohort, $\mathrm{P}=0.040$ by chi-square. Two recent studies reviewing GT trends by collecting retrospective data across multiple hospitals reported no gender differences in infants who received GTs $(\mathrm{n}=333$ and 360). Studies have shown that males are more likely 
to develop BPD and more likely to have more severe BPD and this may explain the sex difference in GT outcome. ${ }^{16}$ Since this finding seems quite variable, it should be examined in larger data sets to assess its reproducibility.

In conclusion, our data show how NICU respiratory course affects achieving full oral feeding. The documentation of respiratory progression during the NICU course, showing improvement in the need for respiratory support from day 30 of age to 32w PMA, and finally 36w PMA, may guide us in finding an opportunity to allow more oral stimulation and feeding during this time. One example, currently being employed at UH Rainbow Babies \& Children's Hospital for older NICU infants with BPD who are on HFNC, is decreasing flow to 2 LPM just for the 20-30 minutes of an oral feeding, which has proven successful thus far (personal communication, Dr. Monika Bhola). The unit policy is to allow oral feeding if an infant is on [?]2 LPM. We are hoping to study this practice as part of a quality improvement project. Are the differences among NICU practices something that can teach us best practice? Our data offers the impetus for those who are experts in oral feeding to help us do better.

\section{References:}

1. Greene NH, Greenberg RG, O'Brien SM, et al. Variation in Gastrostomy Tube Placement in Premature Infants in the United States. Am J Perinatol. 2019;36(12):1243-1249.

2. Jadcherla SR, Bhandari V. "Pressure" to feed the preterm newborn: associated with "positive" outcomes? Pediatr Res.2017;82(6):899-900.

3. Gaon P, Lee S, Hannan S, Ingram D, Milner AD. Assessment of effect of nasal continuous positive pressure on laryngeal opening using fibre optic laryngoscopy. Arch Dis Child Fetal Neonatal Ed.1999;80(3):F230-232.

4. Gehle DB, Chapman A, Gregoski M, Brunswick M, Anderson E, Ramakrishnan V, Muhammad LN, Head W, Lesher AP, Ryan RM/ A Predictive Model for Preterm Babies Born $<30$ Weeks Gestational Age Who Will Not Attain Full Oral Feedings and Require a Surgical Gastrostomy Tube/ Submitted to Journal of Perinatology .

5. Shennan AT, Dunn MS, Ohlsson A, Lennox K, Hoskins EM. Abnormal pulmonary outcomes in premature infants: prediction from oxygen requirement in the neonatal period. Pediatrics.1988;82(4):527-532.

6. Chapman A, George K, Selassie A, Lesher AP, Ryan RM. NICU infants who require a feeding gastrostomy for discharge. J Pediatr Surg.2020.

7. Thebaud B, Goss KN, Laughon M, et al. Bronchopulmonary dysplasia.Nat Rev Dis Primers. 2019;5(1):78.

8. Stoll BJ, Hansen NI, Bell EF, et al. Neonatal outcomes of extremely preterm infants from the NICHD Neonatal Research Network.Pediatrics. 2010;126(3):443-456.

9. Gianni ML, Sannino P, Bezze E, et al. Effect of co-morbidities on the development of oral feeding ability in pre-term infants: a retrospective study. Sci Rep. 2015;5:16603.

10. Lau C, Smith EO, Schanler RJ. Coordination of suck-swallow and swallow respiration in preterm infants. Acta Paediatr.2003;92(6):721-727.

11. Dumpa V, Kamity R, Ferrara L, Akerman M, Hanna N. The effects of oral feeding while on nasal continuous positive airway pressure (NCPAP) in preterm infants. J Perinatol. 2020;40(6):909-915.

12. Mizuno K, Nishida Y, Taki M, et al. Infants with bronchopulmonary dysplasia suckle with weak pressures to maintain breathing during feeding. Pediatrics. 2007;120(4):e1035-1042.

13. Gewolb IH, Bosma JF, Taciak VL, Vice FL. Abnormal developmental patterns of suck and swallow rhythms during feeding in preterm infants with bronchopulmonary dysplasia. Dev Med Child Neurol.2001;43(7):454-459. 
14. Hanin M, Nuthakki S, Malkar MB, Jadcherla SR. Safety and Efficacy of Oral Feeding in Infants with BPD on Nasal CPAP. Dysphagia.2015;30(2):121-127.

15. Tubbs-Cooley HL, Pickler, R.H., Meinzen-Derr, J.K. . Missed oral feeding opportunities and preterm infants' time to achieve full oral feedings and neonatal intensive care unit discharge. Am J Perinatol. 2015;32(1):1-8.

16. Collaco JM, Aherrera AD, McGrath-Morrow SA. The Influence of Gender on Respiratory Outcomes in Children With Bronchopulmonary Dysplasia During the First 3 Years of Life. Pediatr Pulmonol.2017;52(2):217-224.

\section{Hosted file}

Respiratory Tables Final.docx available at https://authorea.com/users/421763/articles/527653severity-of-respiratory-disease-is-correlated-with-time-of-first-oral-feeding-and-needfor-a-gastrostomy-tube-at-discharge-in-premature-infants-born-at-30-weeks-of-gestation

Mean FiO2 for G-tube vs Non-G-tube Babies

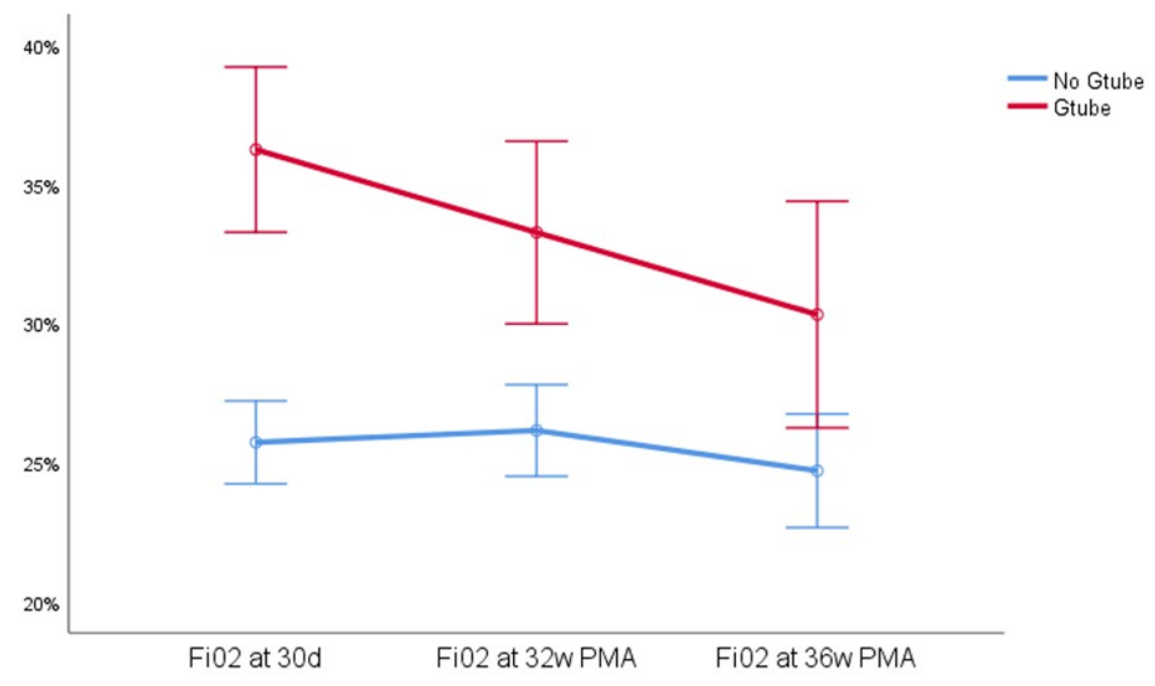

Error bars: $95 \% \mathrm{Cl}$ 
Figure 2A

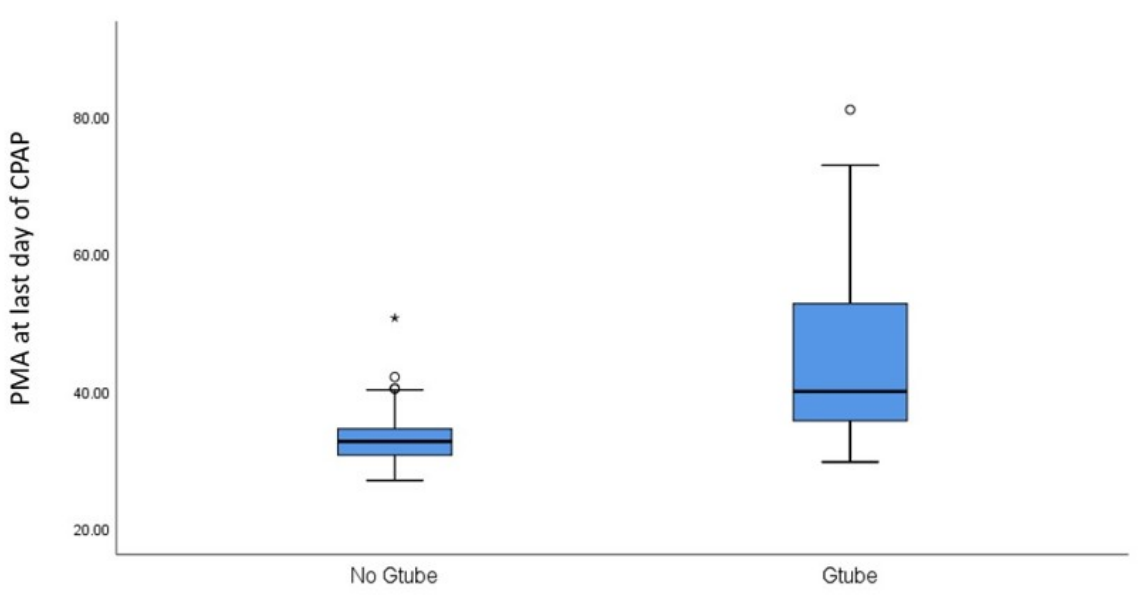

Figure 2B

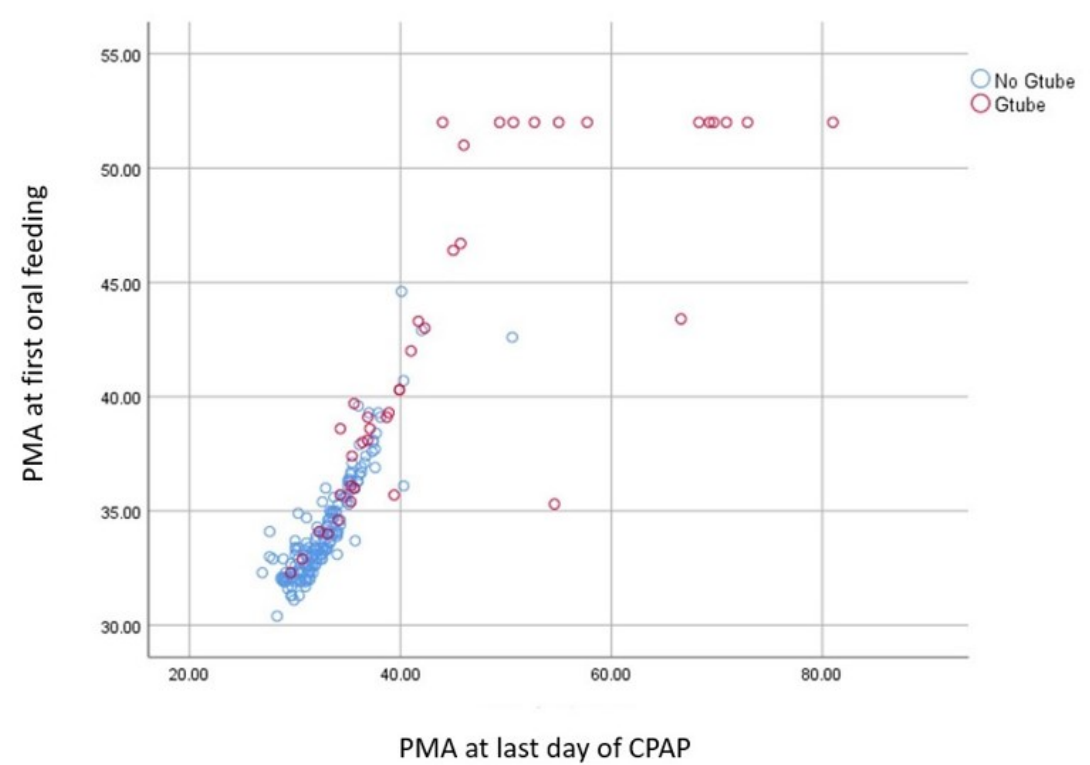




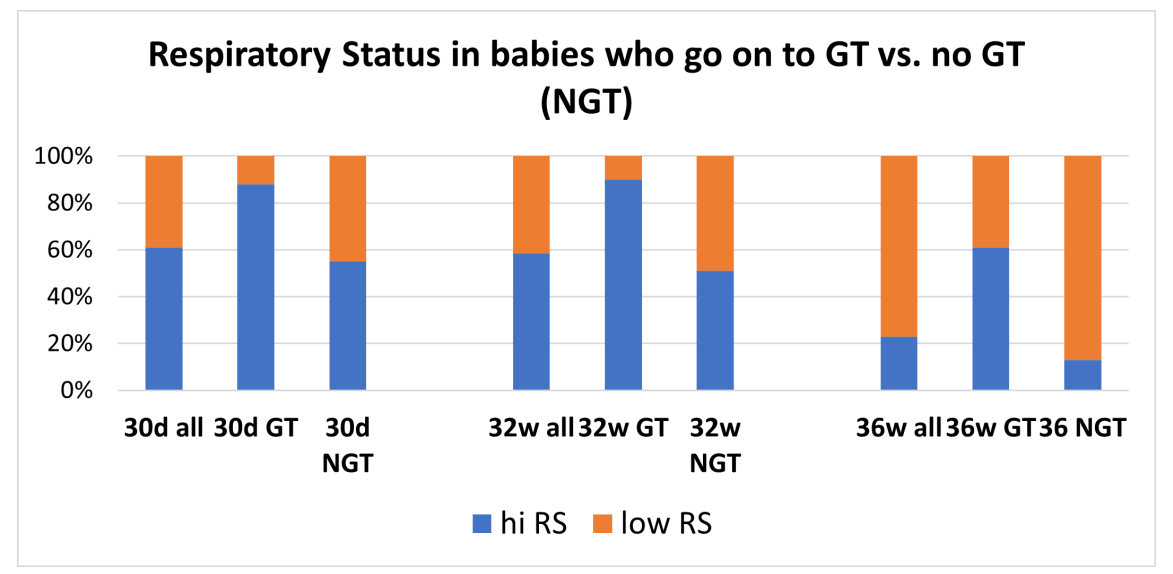

Figure 4A

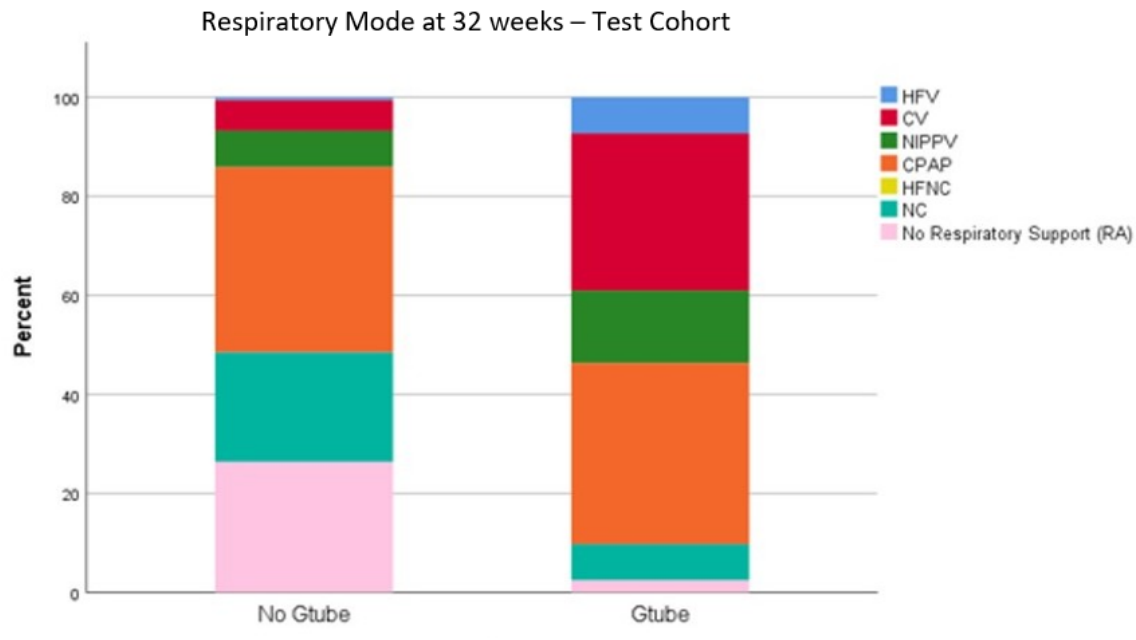

Figure $4 \mathrm{~B}$

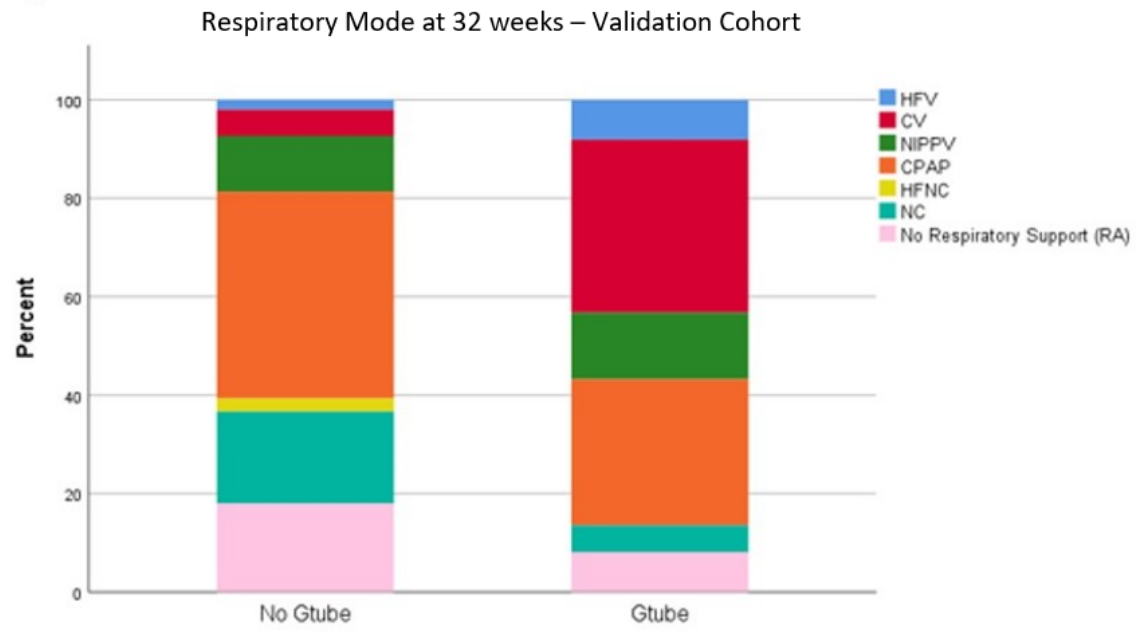

